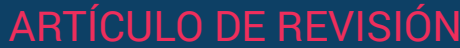

\section{Legionella pneumophila: un patógeno emergente en Argentina}

\author{
Recibido: 5/7/21 Aceptado: 22/9/21
}

Marcelo Gabriel Medina' ${ }^{1}$, Liliana Silvina Lösch²@ Luis Antonio Merino ${ }^{3}$. $^{-}$

\section{RESUMEN}

La enfermedad del Legionario es causada por bacterias pertenecientes al género Legionella, siendo la especie pneumophila el principal agente etiológico de esta patología. Esta bacteria se describió por primera vez en 1977 como causa de un brote de neumonía grave registrado en 1976 en un centro de convenciones en los Estados Unidos de América. La enfermedad se presenta como una neumonía atípica, responsable del 1 al $15 \%$ de los casos de neumonías adquiridas en la comunidad (NAC), del 5 al $10 \%$ de neumonías del adulto y del $1 \%$ en menores de 15 años. Los miembros de la familia Legionellaceae son bacilos aeróbicos gramnegativos que crecen lentamente y se encuentran ampliamente distribuidos en cuerpos de agua. La forma más común de transmisión de Legionella spp es la inhalación de aerosoles contaminados generados a partir de fuentes de agua artificiales. Se asocian con la aparición de brotes esporádicos y epidémicos en la comunidad y en infecciones nosocomiales. Las especies pertenecientes al género Legionella se consideran patógenos emergentes transmitidos por el agua.

El objetivo de este trabajo es realizar una revisión sobre las manifestaciones y presentaciones clínicas de la infección causada por $L$. pneumophila, en virtud de que es considerado mundialmente un patógeno emergente y por existir evidencias de su presencia en sistemas de almacenamiento de agua tratada en la región nordeste de la República Argentina, razón primordial para alertar y actualizar conocimientos al respecto.

Palabras clave: Legionella pneumophila, fuentes ambientales, patógeno emergente transmitido por agua.
' Mgter. Área de Medicina Tropical. Instituto de Medicina Regional. Universidad Nacional del Nordeste. Avenida Las Heras 727. CP 3500, Resistencia,Chaco. drmarcelomedina@gmail.com.

${ }^{2}$ Dra. Área de Bacteriología. Instituto de Medicina Regional, Universidad Nacional del Nordeste. Facultad de Medicina. Universidad Nacional del Nordeste. Avenida Las Heras 727. CP 3500, Resistencia, Chaco. silvinalosch@gmail.com.

${ }^{3}$ Dr. Área de Bacteriología. Instituto de Medicina Regional, Universidad Nacional del Nordeste. Código Avenida Las Heras 727. CP 3500, Resistencia, Chaco. luisantoniomerino@gmail.com.

Autor para correspondencia: Dra. Liliana Silvina Lösch. Área de Bacteriología. Instituto de Medicina Regional, Universidad Nacional del Nordeste. Facultad de Medicina. Universidad Nacional del Nordeste. Código ORCID: https://orcid.org/0000-0002-5096-5578. Avenida Las Heras 727. CP 3500, Resistencia, Chaco. silvinalosch@gmail.com.

Investigación financiada por la Secretaría General de Ciencia y Técnica de la Universidad Nacional del Nordeste. PI16/L004.

Los autores declaran no tener conflicto de intereses. 


\section{Introducción}

La bacteria Legionella pneumophila se describió por primera vez en 1977 como causa de un brote de neumonía grave registrado en 1976 en los Estados Unidos (1). Desde entonces, se la ha asociado a brotes relacionados con sistemas hídricos artificiales de conducción y almacenamiento de agua deficientemente mantenidos, como las torres de refrigeración, los condensadores de evaporación utilizados para sistemas de acondicionamiento de aire, los sistemas de agua fría y caliente en edificios públicos, privados, nosocomiales y las instalaciones de hidromasajes. El caso particular de neumonías causadas por $L$. longbeachae se relaciona a la exposición a compost y tierras para macetas (2-4).

Lalegionelosispuedetenerpresentacionesclínicasleves o graves que incluyen fiebre hasta algunas neumonías potencialmente mortales; su causa es la exposición a especies del género Legionella presentes en el agua a través de la inhalación de aerosoles contaminados con esta bacteria. La especie pneumophila es la principal causa de infección e incluso de brotes epidémicos. Esta y otras especies de Legionella se encuentran distribuidas mundialmente en lagos, ríos, arroyos, aguas termales y otros sistemas hídricos. Otras especies, incluida L. longbeachae, se pueden hallar en mezclas de tierra para macetas y compost, desde donde también pueden ser causa de infección. A menudo la enfermedad se clasifica como neumonía adquirida en la comunidad (NAC), en viajes o en hospitales, dependiendo del tipo de exposición $(2,5-7)$.

La dosis infectante de este patógeno se desconoce dado que su concentración en la fuente ambiental difiere de la alcanzada en el aire $(8,9)$. La probabilidad de desarrollar la enfermedad depende de una tríada conformada por el medio ambiente (concentración de Legionella en la fuente de agua, de la producción y dispersión de aerosoles), el huésped (edad y enfermedades preexistentes) y la virulencia de la cepa particular de Legionella que se trate. La mayoría de las infecciones no causan enfermedad (7-9).

\section{Desarrollo}

\section{Agente causal de legionelosis}

Legionella es el único género de la familia Legionellaceae, conformado por 58 especies y aproximadamente 70 serotipos diferentes, todas ellas recuperadas de ambientes acuáticos y de las cuales 30 pueden causar enfermedad humana. No obstante, la legionelosis es causada principalmente por Legionella pneumophila, conformada por al menos 14 serogrupos diferentes, siendo los grupos 1, 4 y 7 los que están implicados con mayor frecuencia en las infecciones en humanos. Sin embargo, en Australia y Nueva Zelanda la especie más frecuente asociada a neumonías es $L$. longbeachae. Las otras especies comunmente identificadas en la enfermedad humana son Legionella micdadei, L. bozemanae, L. feeleii y L. dumofii $(2,8,10)$.

Legionella spp posee una distribución mundial. Se encuentra en ambientes naturales de agua dulce e incluso en aguas marinas bajo ciertas condiciones de salinidad y temperatura $(11,12)$. No obstante, el riesgo mayor se encuentra en los sistemas de ingeniería construidos por el hombre, como lo son los sistemas de refrigeración, distribución y almacenamiento de agua. Las bacterias viven y crecen en medios acuáticos a temperaturas comprendidas entre los $20^{\circ} \mathrm{C}$ y los $50^{\circ} \mathrm{C}$ (temperatura óptima: $35^{\circ} \mathrm{C}$ ) y pueden sobrevivir y proliferar parasitando protozoos libres o dentro de los biofilms que se forman en los sistemas de agua $(4,13,14)$. En los ambientes con bajo contenido de nutrientes, Legionella spp puede encontrarse bajo la forma de viable no cultivable (VNC). La infección de células humanas, principalmente macrófagos, se realiza por medio de un mecanismo similar al que utilizan para infectar protozoos $(9,15,16)$.

\section{Transmisión}

La forma de transmisión más frecuente de Legionella es la inhalación de aerosoles generados a partir de fuentes contaminadas, como torres de enfriamiento para aire acondicionado, sistemas de agua fría y caliente, humidificadores e instalaciones de hidromasajes. También, aunque mucho menos frecuente, por microaspiración de agua contaminada o por aplicación directa (7). En los últimos años también se identificó a las plantas de tratamiento de efluentes domésticos como causales de brotes. El tamaño de las partículas del aerosol debe ser inferior a las $10 \mu \mathrm{m}$ para que se deposite a nivel alveolar $(9,17,18)$.

A la fecha, solo se reporta un caso de probable transmisión de persona a persona de Legionella spp $(10,19)$. En pacientes inmunocomprometidos se pueden presentar formas de trasmisión poco frecuentes de esta bacteria, como lo son a través de la piel o por exposición durante intervenciones quirúrgicas $(9,20)$. 


\section{Incidencia}

La legionelosis es un importante problema de salud pública, pero en la actualidad la incidencia global de esta enfermedad es desconocida principalmente por la falta de diagnóstico y los subrregistros. A pesar de ello, la información disponible mejoró en los últimos años con el aumento del diagnóstico y la notificación de casos. Legionelosis es de declaración obligatoria en los Estados Unidos, Canadá, Singapur, Unión Europea, Israel, Japón, Australia y Nueva Zelanda. En la Argentina, Legionella pneumophila forma parte de los agentes que deben ser notificados como causales de infecciones respiratorias bacterianas a través del Sistema de Vigilancia de Laboratorio (SIVILA) $(10,21)$.

Las infecciones por este microorganismo registran un aumento de casos en los últimos años y han originado brotes tanto en la comunidad como asociados a hospitales y hoteles, principalmente en Estados Unidos y Europa. El Centro para el Control y Prevención de Enfermedades de los Estados Unidos (CDC) reporta aproximadamente 5000 casos de enfermedad del legionario por año. En 2016 y 2017 se reportaron 6141 y 7458 casos respectivamente. Asimismo, en el período comprendido entre 2000 y 2014 el incremento de casos de legionelosis fue del $286 \%$ (10, 22, 23). El Centro Europeo para la Prevención y el Control de las Enfermedades (ECDC) reportó un incremento de casos confirmados del $65 \%$, de 6882 a 10.672 durante el período 2014-2018. En 2018, Italia, Francia, España, Alemania, Países Bajos y Reino Unido tuvieron más de 9100 reportes (24). Las posibles razones de este aumento de casos se atribuyen a: 1) un aumento continuo en el desarrollo de los sistemas de ingeniería de agua adecuados para el desarrollo de Legionella, como las instalaciones de refrigeración; 2) el envejecimiento de la sociedad en los países desarrollados; y 3 ) un mejor diagnóstico y reporte (8).

En Latinoamérica en general y en la Argentina en particular existe poca información de la incidencia de Legionella spp, situación posiblemente atribuible a la presentación en forma de casos esporádicos y no de brotes. En la Argentina se reporta una incidencia del $2 \%$ de todas las neumonías $(25,26)$. Los reportes publicados en nuestro país provienen de lo presentado por Lopardo y col. (2002), correspondiente a un estudio prospectivo realizado en la Clínica y Maternidad Suizo-Argentina y en la Clínica Finochietto, y por Luna y col. (2004) sobre un estudio retrospectivo realizado el Hospital de Clínicas
José de San Martín de la Universidad de Buenos Aires, y el correspondiente al Ministerio de Salud de la Provincia de Buenos Aires en relación al brote por Legionella spp en el Hospital Nuestra Sra. del Carmen de la localidad de Carmen de Areco en 2013 (27-29). En 2015, Mykietiuk y col. exponen los resultados de un estudio multicéntrico nacional donde se evaluó la prevalencia de Legionella spp como agente causal de NAC y donde la especie pneumophila se presenta como la tercera causa de esta enfermedad, al mismo tiempo que se informa el primer aislamiento por cultivo de esta bacteria en el país. En 2019, Giordano y col. muestran que en un estudio realizado en Córdoba el 4,4\% de los casos de NAC fueron causados por este patógeno, utilizando antígeno urinario como metodología diagnóstica $(30,31)$. Todos los trabajos citados confirman a Legionella pneumophila como causa de NAC en nuestro medio. En los pacientes con NAC grave se deben maximizar los esfuerzos por descartar a Legionella spp como causal de esta enfermedad.

Los factores de riesgo del huésped para la enfermedad del legionario incluyen sexo masculino, edad mayor de 50 años, tabaquismo, abuso de alcohol, diabetes, enfermedades respiratorias o renales crónicas, neoplasias hematológicas e inmunosupresión $(10,32)$. Los factores de riesgo para la neumonía nosocomial incluyen: intervención quirúrgica reciente, intubación, ventilación mecánica, aspiración, presencia de sondas nasogástricas y utilización de equipo de terapia respiratoria. Los huéspedes más susceptibles a esta bacteria son los pacientes inmunocomprometidos. Entre los factores que aumentan la mortalidad por legionelosis se encuentran retraso en el diagnóstico y en la administración de un tratamiento antibiótico adecuado y la presencia de otras enfermedades $(7,10,33)$. Finalmente, en pacientes inmunodeprimidos y mayores de 50 años la enfermedad del legionario es una causa frecuente de neumonía, puede provocar cavitación y empiema como así también manifestaciones extrapulmonares de la enfermedad (34).

\section{Clínica}

La infección por Legionella, legionelosis, posee dos presentaciones clínicas: una forma no neumónica y la forma neumónica.

La forma no neumónica (fiebre de Pontiac) es una enfermedad aguda, autolimitada, de resolución espontánea, que se manifiesta con síntomas similares a los de la gripe y remite generalmente al cabo de 2 a 5 días. 
El periodo de incubación oscila entre unas pocas horas y un máximo de 48 horas. Los principales síntomas son fiebre, escalofríos, cefalea, malestar general y mialgia. No necesita intervención médica ni tratamiento antibiótico y no se conocen casos de muerte asociados a este tipo de infección $(7,10,32,33)$.

La forma neumónica de la enfermedad del legionario tiene, en general, un periodo de incubación de 2 a 10 días. Los síntomas iníciales son fiebre de hasta $40^{\circ} \mathrm{C}$, pérdida de apetito, cefalea, malestar general, dolor osteo-muscular, dolor de espalda, disnea, dolor torácico, adenopatías, dolor en punta de costado, letargo y presencia de tos seca leve inicial, aunque un $50 \%$ de los pacientes presentan tos productiva y un tercio de ellos tiene hemoptisis. Algunos pacientes también refieren diarrea y confusión. La enfermedad del legionario generalmente se agrava en la primera semana si no se trata. Las complicaciones más frecuentes son insuficiencia respiratoria, conmoción, falla renal aguda y multiorgánica. Sin embargo, se pueden presentar otras formas clínicas menos frecuentes, como neumonía de Pittsburg, sinusitis,celulitis, pancreatitis, pericarditis y endocarditis, entre otras $(7,10,32,33)$. La gravedad de la enfermedad va desde una tos leve hasta una neumonía con rápido desenlace fatal. La muerte sobreviene por neumonía progresiva acompañada de insuficiencia respiratoria y/o conmoción y falla multiorgánica $(10,33)$.

La mayoría de los casos de la enfermedad del legionario es causada por la especie de $L$. pneumophila serogrupo 1 , aunque también pueden aislarse otras como L. bozemanii, L. longbeachae y $L$. micdadei. La curación depende del diagnóstico temprano e implantación del tratamiento antibiótico y suele ser completa, aunque puede requerir varias semanas o meses. En casos raros, la neumonía grave no tratada puede causar daño cerebral $(2,5,35)$.

La incidencia de legionelosis en pacientes $\mathrm{VIH}$ positivo es similar a la población VIH negativa, sin embargo, en el primer grupo la enfermedad suele ser más grave (7). En el caso de los pacientes VIH positivo con neumonía, el estudio realizado por Head y col. demostró la coinfección de Mycobacterium tuberculosis o Pneumocystis jirovecii con diferentes especies de Legionella, lográndose el diagnóstico a partir de técnicas moleculares. En los pacientes coinfectados por Legionella spp la presentación clínica fue más severa (36).

La eritromicina fue el antibiótico de elección para el tratamiento de neumonía por Legionella desde 1976.
Sin embargo, los nuevos antimicrobianos como las fluoroquinolonas y macrólidos, entre ellos levofloxacina, claritromicina y azitromicina, han demostrado una mayor eficacia al disminuir los tiempos de estadía en los centros de salud y la mortalidad (37). La terapia con antibióticos para la enfermedad del legionario requiere de la actividad del compuesto contra esta bacteria y al mismo tiempo alcanzar altas concentraciones del fármaco en los macrófagos alveolares. Entre los antibióticos utilizados para la enfermedad del legionario se incluye doxiciclina, quinolonas y azitromicina. Alternativamente, también son eficaces tigeciclina, trimetoprima sulfametoxazol y rifamipicinas. La duración del tratamiento antimicrobiano no debe ser inferior a los 14 días. Cuando se desconoce el patógeno, la terapia empírica para la neumonía adquirida en la comunidad debe incluir un ß-lactámico más un antibiótico anti-Legionella $(37,38)$.

La tasa de mortalidad por la forma neumónica de la legionelosis depende de la gravedad de la enfermedad, la implementación de un tratamiento antibiótico precoz y eficaz, sumado a diversos factores relacionados con el huésped. Por lo tanto, la tasa de mortalidad oscila alrededor del $10 \%$ y se incrementa a más del $25 \%$ en el caso de pacientes hospitalizados e inmunocomprometidos. Actualmente, no hay ninguna vacuna disponible contra la legionelosis $(7,10,33)$.

El cultivo de muestras del tracto respiratorio inferior es la técnica de referencia para el diagnóstico de legionelosis, el cual se debe realizar en la fase aguda de la infección y previo a la instauración de tratamiento antibiótico. El medio de cultivo utilizado es el al agar BCYE (Buffered Charcoal Yeast Extract), suplementado con antibióticos y cisteína. Las muestras deben ser tratadas previamente con solución ácida o calor para minimizar la microbiota comensal respiratoria $(5,10)$. A partir del cultivo se pueden practicar estudios epidemiológicos, de sensibilidad antimicrobiana y tipificación molecular. Sin embargo, tanto en Estados Unidos como en Europa solo el $5 \%$ y $12 \%$ de los casos de legionelosis fueron confirmados por esta técnica. Esta baja sensibilidad del cultivo también es reportada en Argentina por el grupo de Aguerre y col. (10, $22,39)$. La detección del antígeno urinario es una técnica de diagnóstico rápido muy utilizada en la clínica para los casos de legionelosis, sobre todo en los pacientes que no producen esputo suficiente para el cultivo; es igualmente específico pero menos sensible (70\%). Sin embargo, solo está disponible para los casos donde el agente etiológico es $L$. pneumophila serotipo 1. Las infecciones causadas 
por la especie no pneumophila no son diagnosticadas regularmente por las limitaciones de los actuales métodos diagnósticos. Las técnicas moleculares pueden mejorar el diagnóstico de la enfermedad del legionario, dado que detectan cualquier especie y serogrupo $(34,36,40-42)$.

\section{Medidas de prevención}

La prevención de la enfermedad del legionario requiere de la aplicación de medidas de control que minimicen la colonización y proliferación de Legionella spp en los sistemas de distribución, almacenamiento de agua, en los de refrigeración y la generación de aerosoles a partir de esas fuentes. Las autoridades o responsables de los edificios y de los sistemas de abastecimiento de agua son los encargados de garantizar la calidad del agua, para lo cual se requiere seguimiento periódico de las medidas de control de los riesgos identificados, incluida la presencia de Legionella (35). En reservorios domiciliarios de agua de la ciudad de Resistencia, Chaco, se determinó un 34,9\% de muestras positivas para L. pneumophila (43). Si bien no siempre resulta posible erradicar la fuente de infección, sí se pueden reducir sustancialmente los riesgos (44).

Algunas medidas a aplicar son el mantenimiento de las instalaciones y aparatos, en particular mediante su limpieza y desinfección sistemáticas y la aplicación de métodos físicos y/o químicos para limitar al máximo la proliferación de esta bacteria. Para ello, la Organización Mundial de la Salud recomienda $(32,35)$ :

- Mantener, limpiar y desinfectar periódicamente las torres de enfriamiento, utilizando de manera frecuente o sistemática agentes biocidas.

- Instalar separadores de gotas para reducir la difusión de aerosoles de las torres de enfriamiento.

- Mantener una concentración idónea de agentes biocidas, por ejemplo, cloro en las instalaciones de hidromasaje, asegurando el vaciado y la limpieza completos de todo el sistema por lo menos una vez a la semana.

- Mantener limpios los sistemas de agua fría y caliente, asegurando al mismo tiempo que el agua caliente se mantenga por encima de $50{ }^{\circ} \mathrm{C}$ (lo que significa que debe salir de la unidad de calentamiento a $60^{\circ} \mathrm{C}$ o más) y el agua fría por debajo de $25^{\circ} \mathrm{C}$ o, mejor aún, de $20^{\circ} \mathrm{C}$, o bien tratar las instalaciones con un biocida adecuado para limitar el crecimiento bacteriano, sobre todo en hospitales y otros centros sanitarios y geriátricos.

- Reducir el estancamiento abriendo semanalmente los grifos no utilizados de los edificios.

Todas estas medidas reducirán el riesgo de contaminación e infección $(40,41)$.

\section{Conclusiones}

Es indiscutible que la exposición a aguas contaminadas e inhalación de aerosoles generados a partir de las mismas sea la forma más común de infección por Legionella. Su incidencia en Argentina es baja, con tendencia al aumento, quizás debido a que no se piensa en este microorganismo -o porque se lo considera una causa poco frecuente o inexistente de neumonía adquirida en la comunidad en esta área del mundo-y por falta del uso sistemático de los métodos diagnósticos y/o a la aplicación de tratamientos empíricos para NAC que cubren $L$. pneumophila y que podrían impedir su aislamiento e identificación precisa en cultivos. Por lo antes expuesto, esta revisión pretende llamar la atención a los profesionales de la salud en la necesidad de realizar estudios epidemiológicos con pruebas confirmatorias para obtener datos confiables sobre la emergencia real de esta bacteria, cuya presencia en sistemas de agua en la región NEA de Argentina fue confirmada, y que representa un problema grave de salud pública en diferentes partes del mundo. 


\section{Bibliografía}

1. Collier SA, Deng L, Adam EA, Benedict KM, Beshearse EM, Blackstock AJ, et al. Estimate of burden and direct healthcare cost of infectious waterborne disease in the United States. Emerg Infect Dis. 2021;27(1):140-9.

2. Bell H, Chintalapati S, Patel P, Halim A, Kithas A, Schmalzle SA. Legionella longbeachae pneumonia: Case report and review of reported cases in nonendemic countries. IDCases. 2021;23:e01050.

3. Paranjape K, Bédard É, Whyte LG, Ronholm J, Prévost M, Faucher SP. Presence of Legionella spp. in cooling towers: the role of microbial diversity, Pseudomonas, and continuous chlorine application. Water Res. 2020;169:1-11.

4. DilgerT,MelzlH,Gessner A. Legionellacontamination in warm water systems: A species-level survey. Int J Hyg Environ Health. 2018;221(2):199-210.

5. Ausina V, Vicente C, Cercenado E, Antolín CP. Diagonstico microbiológico y control de Legionelosis. Cercenado Emilia CR, editor. Sociedad Española de Enfermedades Infecciosas y Microbiología Clínica; 2005. (Procedimientos en Microbiología Clínica). Report No.: 20.

6. Whiley $\mathrm{H}$, Bentham R. Legionella longbeachae and Legionellosis. Emerg Infect Dis. 2011;17(4):579-83.

7. VaquéRafartJ,MartínezGómezX. Epidemiologíadela legionelosis. Med Clin (Barc). 2002;1 19(suplemento 2):14-24.

8. Kirschner AKT. Determination of viable legionellae in engineered water systems: Do we find what we are looking for? Water Res. 2016;93:276-88.

9. Prussin AJ, Schwake DO, Marr LC. Ten questions concerning the aerosolization and transmission of Legionella in the built environment. Build Environ. 2017;123:684-95.

10. Burillo A, Pedro-Botet ML, Bouza E. Microbiology and Epidemiology of Legionnaire's Disease. Infect Dis Clin North Am. 2017;31(1):7-27.

11. Linsak DT, Kese D, Broznic D, Lusic DV, Cenov A, Moric M, et al. Sea water whirlpool spa as a source of Legionella infection. J Water Health. 2021; In press:1-12.

12. Grúas C, Llambi S, Arruga MV. Detection of Legionella spp. and Legionella pneumophila in water samples of Spain by specific real-time PCR. Arch Microbiol. 2014;196(1):63-71.

13. Wingender $\mathrm{J}$, Flemming $\mathrm{HC}$. Biofilms in drinking water and their role as reservoir for pathogens. Int J Hyg Environ Health. 2011;214(6):417-23.
14. Kruse EB, Wehner A, Wisplinghoff $H$. Prevalence and distribution of Legionella spp in potable water systems in Germany, risk factors associated with contamination, and effectiveness of thermal disinfection. Am J Infect Control. 2015;44(4):470-4.

15. Cervero-Aragó $S$, Schrammel B, Dietersdorfer $E$, Sommer R, Lück C, Walochnik J, et al. Viability and infectivity of viable but nonculturable Legionella pneumophila strains induced at high temperatures. Water Res. 2019;158:268-79.

16. Dietersdorfer E, Kirschner A, Schrammel B, Ohradanova-Repic A, Stockinger $\mathrm{H}$, Sommer R, et al. Starved viable but non-culturable (VBNC) Legionella strains can infect and replicate in amoebae and human macrophages. Water Res. 2018;141:428-38.

17. Buse HY, Schoen ME, Ashbolt NJ. Legionellae in engineered systems and use of quantitative microbial risk assessment to predict exposure. Water Res. 2012;46(4):921-33.

18. Caicedo C, Rosenwinkel $\mathrm{KH}$, Exner $\mathrm{M}$, Verstraete W, Suchenwirth R, Hartemann $P$, et al. Legionella occurrence in municipal and industrial wastewater treatment plants and risks of reclaimed wastewater reuse: Review. Water Res. 2019;149:21-34.

19. Correia AM, Ferreira JS, Borges V, Nunes A, Gomes $B$, Capucho R, et al. Probable Person-to-Person Transmission of Legionnaires' Disease. N Engl J Med. 2016;374(5):497-8.

20. Padrnos LJ, Blair JE, Kusne S, Dicaudo DJ, Mikhael JR. Cutaneous legionellosis: Case report and review of the medical literature. Transpl Infect Dis. 2014;16(2):307-14.

21. Dirección de Epidemiología. Ministerio de Salud de la Nación. Abordaje Integral de las Infecciones Respiratorias Agudas. Guía para el equipo de Salud. 2010.

22. Deven AB, Priti L, Tripathi S, Cooley L, Lee S, Smith J, et al. Legionnaire's Disease Surveillance Summary Report, United States 2016-2017. Centers for Disease Control and Prevention. 2020.

23. Bonetta S, Bonetta S, Ferretti E, Balocco F, Carraro E. Evaluation of Legionella pneumophila contamination in Italian hotel water systems by quantitative real-time PCR and culture methods. J Appl Microbiol. 2010;108(5):1576-83.

24. European Centre for Disease Prevention and Control. European Centre for Disease Prevention and Control. Legionnaires' disease. In ECDC.Annual epidemiological report for 2018. Stockholm:ECDC; 2020. 2020. 
25. Lopardo G, Basombrio A, Clara L, Desse J, De Vedia L, Di Libero $E$, et al. Neumonía adquirida de la comunidad en adultos. Recomendaciones sobre su atención. Med (Buenos Aires). 2015;75:245-57.

26. Arancibia $F$, Cortes $C P$, Valdés $M$, Cerda J, Hernández A, Soto L, et al. Importance of legionella pneumophila in the etiology of severe communityacquired pneumonia in Santiago, Chile. Chest. 2014; 145(2):290-6.

27. Luna C, Brea Folco J, Aruj P, Rebora K, Balsebre C, Absi R, et al. Neumonía por Legionella pneumophila. Experiencia en un hospital universitario de Buenos Aires. Med (Buenos Aires). 2004;64:97-102.

28. Lopardo G, Sturba E, Martinez M, Roel J, Gamba A, Biondi $\mathrm{H}$, et al. Detección de infección aguda por Legionella pneumophila en pacientes con neumonía adquirida en la comunidad en la ciudad de Buenos Aires. Med (Buenos Aires). 2002;62:145-8.

29. Dirección de Epidemiología de la Provinca de Buenos Aires $\mathrm{M}$ de $\mathrm{S}$ de la $\mathrm{P}$ de BA. Brote de Enfermedad Respiratoria Aguda Grave por Legionella spp en Hospital Nuestra Sra. del Carmen de la Localidad de Carmen de Areco [Internet]. 2013. Available from: https://silo.tips/download/brote-de-enfermedadrespiratoria-aguda-grave-por-legionella-spp-enhospital-nues

30. Giordano G, Abiega C, Vilaro M CJ. Estudio de neumonía adquirida de la comunidad debido a Legionella pneumophila serotipo 1. In: Sociedad Argentina de Infectología, editor. XIX Congreso SADI 2019. San Miguel de Tucumán, Argentina.; 2019.

31. Mykietiuk, Doreski P, Stock A, Luna C, Lopardo G, Gentile J, VillarealS, Nannini E, Alzogaray M, Das A, Clark K, Jamieson B, Keedy K, Oldach D, Fernandes $\mathrm{P}$ SM. Legionella spp. como causa de neumonía adquirida en la comunidad en Argentina. In: Sociedad Argentina de Infectología, editor. XV Congreso SADI 2015. Ciudad Autónoma de Buenos Aires, Argentina.; 2015.

32. Organización Mundial de la Salud. Legionelosis [Internet]. 2018. Available from: https://www.who. int/es/news-room/fact-sheets/detail/legionellosis

33. Sharma L, Losier A, Tolbert T, Dela Cruz CS, Marion CR. Atypical Pneumonia: Updates on Legionella, Chlamydophila, and Mycoplasma Pneumonia. Clin Chest Med. 2017;38(1):45-58.

34. Lanternier F, Ader F, Pilmis B, Catherinot E, Jarraud S, Lortholary O. Legionnaire's Disease in Compromised Hosts. Infect Dis Clin North Am. 2017;31(1):123-35.
35. World Health Organization. Legionella and the prevention of legionellosis. World Heal. World Health Organization, editor. Geneva, Switzerland; 2007. $252 \mathrm{p}$.

36. Head BM, Trajtman A, Bernard K, Burdz T, Vélez $L$, Herrera $M$, et al. Legionella co-infection in HIVassociated pneumonia. Diagn Microbiol Infect Dis. 2019;95(1):71-6.

37. Kato $H$, Hagihara $M$, Asai $N$, Shibata $Y$, Koizumi $Y$, Yamagishi $Y$, et al. Meta-analysis of fluoroquinolones versus macrolides for treatment of legionella pneumonia. J Infect Chemother. 2021;27(3):424-33.

38. Cunha CB, Cunha BA. Antimicrobial Therapy for Legionnaire's Disease: Antibiotic Stewardship Implications. Infect Dis Clin North Am. 2017;31(1):179-91.

39. Aguerre L, Martínez C, Rocca MF, Cipolla L, Armitano $\mathrm{R}$, Prieto $\mathrm{M}$. Legionellosis in Argentina. In: International Journal of Infectious Diseases. International Society for Infectious Diseases; 2018. p. 150.

40. World Health Organization. Guidelines for drinkingwater quality: fourth edition incorporating the first addendum. $4^{\circ}$. Ginebra, Suiza: World Health Organization; 2017. $380 \mathrm{p}$.

41. Meier T, Bendinger B. Survival of pathogens in drinking water plumbing systems: Impact factors and sanitation options. Water Sci Technol Water Supply. 2016;16(4):931-41.

42. Benitez AJ, Winchell JM. Rapid detection and typing of pathogenic nonpneumophila Legionella spp. Isolates using a multiplex real-time PCR assay. Diagn Microbiol Infect Dis. 2016;84(4):298-303.

43. Lösch LS, Deluca GD, Medina MG, Yarros A, Weber $M$, Merino LA. Presencia de especies de Legionella en reservorios domiciliarios de agua de Resistencia Chaco, Argentina. Rev Argentina Salud Pública. 2019;10(40):19-25.

44. Gea-Izquierdo E, Mezones-Holguin E, Haro-Garcia L. Acciones de prevención y control de la legionelosis: un reto para la salud pública española. Rev Peru Med Exp Salud Publica. 2012;29(2):272-6. 
Legionella pneumophila: an emerging pathogen in Argentina

Legionnaires' disease is caused by bacteria belonging to the genus Legionella, being the pneumophila specie the main etiological agent of this pathology. This bacterium was first described in 1977 as the cause of a severe pneumonia outbreak in 1976 at a convention center in the United States of America. The disease presents as an atypical pneumonia, responsible for $1 \%$ to $15 \%$ of cases of community-acquired pneumonia (CAP), $5 \%$ to $10 \%$ of pneumonia in adults and $1 \%$ in children under 15 years of age. Members of the Legionellaceae family are aerobic, gram-negative rods that grow slowly and are widely distributed in water bodies. The most common way of transmission of Legionella spp is the inhalation of contaminated aerosols generated from artificial water sources. They are associated with the appearance of sporadic and epidemic outbreaks in the community and in nosocomial infections. Species belonging to the genus Legionella are considered emerging waterborne pathogens.

The aim of this work is to carry out a review on the manifestations and clinical presentations of the infection caused by $L$. pneumophila, due to that it is considered an emerging pathogen worldwide and because there is evidence of its presence in storage systems of treated water in the Northeast region of the Argentine Republic, primary reason to alert and update knowledge in this regard.

Keywords: Legionella pneumophila, environmental sources, emerging waterborne pathogen. 